\title{
Sistema reprodutivo e polinização de Senna multijuga (Fabaceae) em Mata Atlântica Montana ${ }^{1}$
}

\author{
Breeding system and pollination of Senna multijuga (Fabaceae) \\ in a montane Atlantic Forest
}

Marina Wolowski ${ }^{2} \&$ Leandro Freitas ${ }^{3}$

\begin{abstract}
Resumo
O conhecimento da biologia reprodutiva e polinização de árvores neotropicais vêm aumentando nas últimas décadas. No entanto, ainda é incipiente para a Mata Atlântica e muitas questões persistem, por exemplo, sobre a prevalência de autoincompatibilidade. A biologia floral, o sistema reprodutivo e os visitantes florais de Senna multijuga foram estudados, com o objetivo de verificar a ocorrência de autoincompatibilidade e determinar seus polinizadores. A espécie apresenta características florais típicas do gênero, como flores assimétricas, enantiostilia, heteranteria e deiscência poricida. A antese é diurna e a viabilidade do pólen não diferiu entre os morfos de anteras. Os polinizadores foram abelhas dos gêneros Bombus, Epicharis, Centris e Xylocopa e abelhas menores atuaram como pilhadores de pólen. A reprodução sexual em S. multijuga depende da transferência de pólen pelos polinizadores, pois não foram formados frutos por apomixia ou autopolinização. Este último resultado pode ser devido à depressão por endogamia ou a um mecanismo de autoincompatibilidade de ação tardia, uma vez que houve aborto uniforme de pistilos e penetração de tubos polínicos nos óvulos após autopolinização. Diferença na fecundidade após polinização manual e em condições naturais indica que a limitação polínica afeta o sucesso reprodutivo feminino, assim como a disponibilidade de recursos.

Palavras-chave: autoincompatibilidade de ação tardia, depressão por endogamia, flor de pólen, polinização.
\end{abstract}

\section{Abstract}

Knowledge of reproductive biology and pollination of Neotropical tree species has been increasing in recent decades. However, this is still incipient in the Atlantic Forest, and many questions remain, for instance, about the prevalence of self-incompatibility. We studied the floral biology, breeding system and floral visitors of Senna multijuga in order to verify the occurrence of self-incompatibility and determine its pollinators. This species bears the typical floral traits of the genus, such as asymmetry, enantiostily, heteranthery and poricid anthers. Anthesis is diurnal and pollen viability did not differ among the morphs of anthers. Pollinators were bees of the genera Bombus, Epicharis, Centris and Xylocopa and smaller bees acted as pollen thieves. Sexual reproduction of Senna multijuga depends on the transfer of pollen by pollinators, as no fruit was formed by either apomixis or self-pollination. The latter result may be due to inbreeding depression or by a mechanism of late-acting selfincompatibility, as we observed after self-pollination both penetration of pollen tubes in the ovules and uniform abortion of pistils. Differences on fertility after manual crossings and under natural conditions indicate that female fitness is affected by pollen limitation, as well as by the availability of resources.

Key words: inbreeding depression, late-acting self-incompatibility, pollen flower, pollination.

\section{Introdução}

O conhecimento acerca da biologia reprodutiva e da polinização de espécies arbóreas neotropicais vêm aumentando ao longo das últimas três décadas, a partir dos estudos pioneiros para conjunto de espécies na América Central e do Sul (Bawa 1974; Zapata \& Arroyo 1978; Bawa et al. 1985a,b) e pelo acúmulo de informações de diversos estudos pontuais (e.g. Gibbs \& Sassaki 1998; Gribel et al. 1999; Dick 2001; Freitas \& Oliveira 2002; Carvalho \& Oliveira

${ }^{1}$ Parte da dissertação de Mestrado da primeira autora.

${ }^{2}$ Programa de Pós-Graduação em Botânica, Escola Nacional de Botânica Tropical, R. Pacheco Leão 2040, 22460-030, Rio de Janeiro, RJ, Brasil. mtorres@jbrj.gov.br ${ }^{3}$ Instituto de Pesquisas Jardim Botânico do Rio de Janeiro, R. Pacheco Leão 915, 22460-030, Rio de Janeiro, RJ, Brasil. leandro@jbrj.gov.br 
2003; Quesada et al. 2004). Para espécies arbóreas de Mata Atlântica, os dados ainda são incipientes, embora a quantidade de estudos seja crescente (e.g. Sazima et al. 1985; Goldenberg \& Varassin 2001; Borges et al. 2008; Pires \& Freitas 2008).

As questões que motivaram os primeiros estudos reprodutivos nas florestas tropicais surgiram em torno da alta riqueza de espécies de árvores e suas baixas densidades populacionais, da capacidade dos polinizadores se movimentarem entre as plantas e dos níveis de autopolinização e polinização cruzada nas espécies (Fedorov 1966; Ashton 1969; Janzen 1971). Nesses estudos pioneiros, a autoincompatibilidade foi registrada para a maioria ( $c a .80 \%$ ) das espécies hermafroditas estudadas (Bawa 1974; Bawa et al. 1985a). Alguns autores sugerem que a prevalência da autoincompatibilidade, que implica na necessidade de fecundação cruzada, provavelmente está relacionada à pressões seletivas para a manutenção da variabilidade genética (Bawa 1974; Zapata \& Arroyo 1978; Seavey \& Bawa 1986). Contudo, a autocompatibilidade ocorrente em espécies arbóreas (Sazima et al. 1985; Goldenberg \& Varassin 2001; Pires \& Freitas 2008) pode também ser vantajosa como forma de aumentar a chance de sucesso reprodutivo em espécies cujas populações têm baixa densidade ou em ambientes com escassez de polinizadores.

Em Fabaceae, há uma tendência para maior frequência de sistemas de autoincompatibilidade em grupos de arbóreas que em grupos de herbáceas, e para sistemas de autoincompatibilidade gametofítica (Arroyo 1981). Mais recentemente tem sido registrada autoincompatibilidade de ação tardia (LSI) em várias leguminosas tropicais (Seavey \& Bawa 1986; Gibbs et al. 1999; Lewis \& Gibbs 1999; Carvalho \& Oliveira 2003; Borges et al. 2008). Contudo, nos supostos casos de LSI em que não se identificam barreiras pré-zigóticas, não é fácil distinguir sua ocorrência dos efeitos de depressão por endogamia, isto é, a expressão de alelos recessivos deletérios que impedem o desenvolvimento embrionário (Seavey \& Bawa 1986; Sage et al. 1994; Husband \& Schemske 1996). Por exemplo, em Epilobium obcordatum A.Gray (Onagraceae) foi proposta a ocorrência de LSI pós-zigótica, dada a esterilidade de algumas plantas e aborto de embriões em estágio inicial de desenvolvimento (Seavey \& Bawa 1986). Posteriormente, foi demonstrado que a variação entre a produção de sementes de autopolinização e polinização cruzada nessa espécie era decorrente de depressão por endogamia (Seavey \& Carter 1994). Em algumas espécies de Fabaceae, a formação de progênie predominante alógama também foi associada à depressão por endogamia e não a mecanismos de autoincompatibilidade (Gibbs \& Sassaki 1998; Hufford \&Hamrick2003).

No gênero Senna há registros de autocompatibilidade e autoincompatibilidade de ação tardia (Silva et al. 2002; Carvalho \& Oliveira 2003; Laporta 2005). Especificamente para Senna multijuga (Rich.) Irwin \& Barneby não foi realizado estudo sobre sistema reprodutivo, porém em populações em áreas de pastagem e vegetação secundária, na borda do Parque Estadual do Rio Doce, Minas Gerais, foram observadas taxas de cruzamentos variáveis através de marcadores moleculares, o que sugere ausência de autoincompatibilidade para a espécie (Ribeiro \& Lovato 2004).

Flores assimétricas, enantiostilia, heteranteria e deiscência poricida são características comuns às espécies da subtribo Cassiinae (Fabaceae) (Gottsberger \& Silberbauer-Gottsberger 1988). A enantiostilia evoluiu independentemente em pelo menos dez famílias de angiospermas e normalmente está associada a outras características incluindo a heteranteria, a coleta de pólen por vibração realizada por abelhas, ausência de nectários e a assimetria floral (Barrett et al. 2000; Jesson et al. 2003). A presença desses caracteres em grupos não-relacionados sugere a convergência evolutiva para a qual a posição do polinizador durante a visita é importante para a dispersão do pólen e, consequentemente, para o sucesso reprodutivo masculino (Barrett et al. 2000). A deiscência poricida é comum em espécies em que o pólen é o único recurso floral e a coleta de pólen é ativa, realizada por fêmeas de abelhas capazes de promover vibrações nas peças florais (Gottsberger \& Silberbauer-Gottsberger 1988). Contudo, nem todas as espécies que visitam flores de pólen atuam como polinizadores e a relação de tamanho entre o visitante e o gineceu e os estames longos é uma característica importante para a polinização das espécies do gênero (Gottsberger \& SilberbauerGottsberger 1988; Pinheiro \& Sazima 2007).

Neste estudo são descritos aspectos da biologia floral e do sistema reprodutivo e registrada a composição de visitantes florais de Senna multijuga em uma área de Mata Atlântica, com o objetivo de verificar a existência de mecanismo de autoincompatibilidade e determinar os polinizadores da espécie.

\section{Material e Métodos}

O estudo foi desenvolvido nos anos 2007 e 2008 em área de floresta ombrófila densa montana (sensu Veloso et al. 1991), entre 500 e 1000 m de 
altitude, no Parque Nacional do Itatiaia (PNI) ( $\left.22^{\circ} 27^{\prime} \mathrm{S} ; 4^{\circ} 36^{\prime} \mathrm{W}\right)$. O clima nessa faixa altitudinal é do tipo $C w a$ e $C f b$ (classificação de Köeppen) e apresenta duas estações bem marcadas: um período quente coincidindo com abundantes chuvas e um período frio associado à baixa pluviosidade (Segadas-Vianna \& Dau 1965). Observações e experimentos complementares foram realizados na Área de Proteção Ambiental da Serrinha do

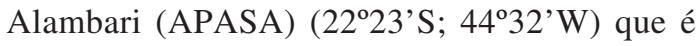
contígua ao PNI e localiza-se na sua zona de amortecimento. Na área de estudo na APASA, a variação altitudinal é semelhante à do PNI e a vegetação se encontra em diferentes estágios de sucessão ecológica.

Senna multijuga (Rich.) Irwin \& Barneby é uma espécie de hábito arbóreo, com 10 a 15 m de altura, que apresenta distribuição ampla na América Central e do Sul, mas com variedades de distribuição geográfica mais restrita (Irwin \& Barneby 1982). Na área de estudo ocorre S. multijuga subsp. lindleyana (Gardner) H.S. Irwin \& Barneby (Morim \& Barroso 2007), a qual se distribui do sul da Bahia ao nordeste de Santa Catarina, nas cristas e declives da Mata Atlântica (Irwin \& Barneby 1982). Senna multijuga é comum entre 50 e $950 \mathrm{~m}$ de altitude, em clareiras, bordas de floresta, matas ciliares e em áreas perturbadas e em regeneração, assim como pode ser preservada em pastagens e plantações para sombrio (Irwin \& Barneby 1982). Também é indicada para restauração de áreas degradadas, como espécie secundária inicial (Moraes et al. 2006), e é cultivada e utilizada para arborização urbana em alguns estados brasileiros (Irwin \& Barneby 1982; Rodrigues et al. 2005). No PNI, a subespécie é frequente na faixa entre 600 e $900 \mathrm{~m}$ de altitude, em locais próximos ao rio Campo Belo, no interior da mata e em áreas de vegetação alterada (Morim 2006; Morim \& Barroso 2007). Na APASA, indivíduos foram observados em bordas de mata, áreas em regeneração e pastagens, bem como nos arredores de residências (Torres 2009). A floração dos indivíduos acompanhados no PNI e na APASA ocorreu entre janeiro e maio e a frutificação entre março e dezembro (Torres 2009). A dispersão das sementes é pelo vento.

A biologia floral foi estudada a partir de material fresco ou estocado em FAA 70\%. A presença de osmósforos foi verificada por meio de teste com vermelho neutro (Dafni 1992). A receptividade do estigma foi determinada utilizando peróxido de hidrogênio (Zeisler 1938), com auxílio de lupa de bolso. A viabilidade polínica foi estimada em 200 grãos de pólen por antera em três anteras de tamanho distintos, corados com carmim acético, com auxílio de microscópio óptico (Dafni 1992). O teste Quiquadrado $\left(X^{2}\right)$ foi utilizado para analisar possíveis diferenças na viabilidade entre os grãos de pólen provenientes desta anteras. O número de grãos de pólen foi estimado em três anteras por tamanho, utilizando a câmara de Neubauer, com auxílio de microscópio óptico. A razão pólen/óvulo foi determinada de acordo com Cruden (1977). Outros aspectos da biologia floral como coloração, odor, horário da antese e duração da flor foram registrados em observações diretas no campo (Dafni 1992).

O sistema reprodutivo foi determinado através de testes de polinização controlados em flores de inflorescências isoladas em sacos de "voile" ainda em botões florais. Foram utilizados três indivíduos em pico de floração em fevereiro e março no PNI e dois indivíduos na APASA em abril de 2008. O acesso às flores foi feito a partir de escada e montagem de andaimes de construção civil ( $c f$. Pires \& Freitas 2008). As flores foram submetidas aos seguintes tratamentos: i. apomixia - flores em pré-antese foram emasculadas e mantidas ensacadas na inflorescência; ii. autopolinização espontânea - flores em pré-antese não manipuladas foram mantidas ensacadas na inflorescência; iii. autopolinização manual - flores em antese em que o conteúdo de pólen de uma antera da própria flor (autogamia) ou de outra flor do mesmo indivíduo (geitonogamia) foi depositado no estigma; iv. polinização cruzada manual - flores em antese em que o conteúdo de pólen de uma antera de outro indivíduo foi depositado no estigma. O número de flores por tratamento consta na tabela 1. Os tratamentos foram distribuídos entre os indivíduos analisados. A autopolinização manual foi aplicada em cinco indivíduos e os demais tratamentos, em quatro. Nos tratamentos autopolinização manual e polinização cruzada, a antera foi dissecada sobre lâmina limpa e o conteúdo depositado no estigma. Nesses tratamentos foram realizados cruzamentos para os três diferentes tamanhos de anteras citados acima.

O crescimento dos tubos polínicos foi verificado utilizando a técnica de fluorescência de Martin (1959) em pistilos provenientes de tratamentos de autopolinização manual e de polinização cruzada manual fixados em FAA 70\% a intervalos de 12, 24, 48 e 72 horas após o tratamento. Ainda, alguns pistilos autopolinizados foram fixados 24 horas após o tratamento e, então, 
desidratados em série etílica ascendente até etanol absoluto e incluídos em hidroxietilmetacrilato (Gerrits \& Smid 1983). Após inclusão e montagem dos blocos, estes foram seccionados longitudinalmente com auxílio de micrótomo de rotação com $3 \mu \mathrm{m}$ de espessura. As secções foram aderidas a lâminas de vidro e coradas com azul de toluidina $0,05 \%$ (O'Brien et al. 1965) e observadas em microscópio óptico de campo claro.

A fim de estimar a produção de frutos em condições naturais, flores em antese foram marcadas e mantidas expostas. O teste Qui-quadrado $\left(X^{2}\right)$ foi utilizado para avaliar possíveis diferenças na produção de frutos formados nos tratamentos (entre cruzamentos com anteras de diferentes tamanhos) e entre os tratamentos polinização cruzada e condições naturais. Os dados de condições naturais são provenientes de um indivíduo do PNI. O outro indivíduo que seria utilizado como controle sofreu forte florivoria por formigas do gênero Atta e não pode ser utilizado para este fim. As flores desse indivíduo que estavam ensacadas não foram danificadas e os dados dos outros tratamentos foram utilizados nas comparações.

Foi feita a contagem de óvulos, sementes desenvolvidas e sementes abortadas de frutos provenientes do tratamento polinização cruzada (18 frutos) e condições naturais (75 frutos) do PNI. A fecundidade foi medida pela razão do número sementes desenvolvidas/número de óvulos. A taxa de aborto foi medida pela razão sementes abortadas/ óvulos. Os frutos foram abertos com pinça e estilete e os óvulos e sementes contados com auxílio da lupa. O número de câmeras seminíferas e cicatrizes de óvulos foram utilizados para estimar o número

Tabela 1 - Produção de frutos (porcentagem do número de frutos/número de flores) após tratamentos de polinização controlados de Senna multijuga no Parque Nacional de Itatiaia e na Área de Proteção Ambiental da Serrinha do Alambari.

Table 1 - Fruit production (percentage of fruit number/flower number) after pollination treatments of Senna multijuga in the Itatiaia National Park and the Environmental Protection Area of the Serrinha Alambari.

\begin{tabular}{lcc}
\hline & $\begin{array}{c}\mathbf{n}^{0} \text { de } \\
\text { flores }\end{array}$ & $\begin{array}{c}\mathbf{n}^{\mathbf{0}} \text { de } \\
\text { frutos (\%) }\end{array}$ \\
\hline Apomixia & 15 & $0(0)$ \\
Autopolinização espontânea & 15 & $0(0)$ \\
Autopolinização manual & 137 & $0(0)$ \\
Polinização cruzada & 104 & $55(53)$ \\
\hline
\end{tabular}

de óvulos. Sementes íntegras, com testa bem formada, foram consideradas desenvolvidas. Sementes com desenvolvimento interrompido e/ou deformadas foram consideradas abortadas. O teste Kruskal-Wallis $(\mathrm{H})$ foi utilizado para analisar possíveis diferenças entre o número de óvulos, de sementes desenvolvidas, de sementes abortadas, a fecundidade e a taxa de aborto dos cruzamentos com anteras de diferentes tamanhos do tratamento polinização cruzada manual. O teste MannWhitnney (U) foi utilizado para analisar possíveis diferenças no número de óvulos, de sementes desenvolvidas, de sementes abortadas, na fecundidade e na taxa de aborto entre os tratamentos polinização cruzada e condições naturais. Os testes estatísticos foram realizados no software STATISTICA 6.0 (Statsoft 2001).

Os visitantes florais foram coletados com puçá e o comportamento de forrageio foi registrado. As observações foram realizadas em dias com céu limpo ou parcialmente encoberto totalizando 12 horas distribuídas entre 8:00 e 17:00h em três indivíduos em fevereiro de 2008 no PNI. As espécies coletadas foram classificadas como polinizadores ou pilhadores com base na observação de contato do corpo da abelha com o estigma, o que se deve ao seu tamanho (Frankie et al. 1983) e comportamento de forrageio (Pinheiro \& Sazima 2007).

\section{Resultados}

As flores de Senna multijuga, dispostas em panículas terminais, são assimétricas e em prato, com 36,81 \pm 2,56 mm (média \pm desvio-padrão, em todo o texto) $(\mathrm{n}=10)$ de diâmetro. As sépalas amarelas, em número de cinco, são livres. As pétalas, também de coloração amarela e em mesma quantidade, são desiguais (Fig. 1a). O androceu é constituído por dois estames grandes, um estame médio, quatro estames pequenos e três estaminóides (Fig. 1a). Os estames grandes apresentam anteras curvas $(7,50 \pm 0,74 \mathrm{~mm}, \mathrm{n}=10)$ e filete alongado $(6,28 \pm 0,94 \mathrm{~mm}, \mathrm{n}=10)$. O estame médio apresenta antera curva $(6,40 \pm 1,51 \mathrm{~mm}, \mathrm{n}=10)$ e filete curto $(2,08 \pm 0,45 \mathrm{~mm}, \mathrm{n}=10)$. Os estames pequenos apresentam anteras retas $(4,56 \pm 0,65 \mathrm{~mm}, \mathrm{n}=10) \mathrm{e}$ filetes curtos $(1,98 \pm 0,20 \mathrm{~mm}, \mathrm{n}=10)$. As anteras apresentam deiscência poricida e o pólen é o único recurso floral. As anteras reagiram ao teste com vermelho neutro e a presença dos osmóforos foi verificada da base ao ápice da antera em estruturas definidas como frisos longitudinais, os quais são constituídos por um maciço de células de origem epidérmica e natureza 
secretora (sensu Guimarães et al. 1984). O pistilo, com $13,93 \pm 0,44 \mathrm{~mm}(\mathrm{n}=10)$ de comprimento (da base ao estigma), apresenta ovário súpero e unilocular, com 45,07 $\pm 6,14(n=75)$ óvulos de placentação sutural. $O$ estigma é circular, em forma de câmara e a abertura está voltada para a parte interna da flor.

A viabilidade dos grãos de pólen foi de 96,33 $\pm 0,02 \%(\mathrm{n}=3)$ nas anteras pequenas, $98,17 \pm 0,003 \%$ $(\mathrm{n}=3)$ para as anteras médias e 97,33 $\pm 0,02 \%(\mathrm{n}=3)$ para as anteras grandes. Não houve diferença para viabilidade polínica do pólen proveniente dos três diferentes tamanhos de antera $\left(X^{2}=3,82, p=0,15\right)$. Anteras pequenas possuíam $11.789 \pm 3.206$ grãos de pólen, anteras médias $12.300 \pm 2.909$ e anteras grandes $20.178 \pm 3.384$. Desta forma, o número total médio de grãos de pólen em uma flor foi estimado em 99.811 e a razão pólen/óvulo em 2.218.

Durante o desenvolvimento do botão floral, o pistilo desloca-se da posição central para posição lateral, à esquerda ou à direita, caracterizando a enantiostilia. Flores de morfos opostos foram encontradas na mesma inflorescência (Fig. 1b). Os estames de anteras grandes acompanham o deslocamento do pistilo no sentido oposto, se aproximando da pétala maior e a abertura do poro é direcionada no sentido da curvatura da pétala maior. O estame médio acompanha o movimento do pistilo e fica em posição oposta à pétala maior e o poro da antera é extrorso (Fig. 1a). Os estames pequenos se dispõem no centro da flor e a abertura dos poros também é direcionada ao centro da flor. Durante o deslocamento do pistilo e estames, a corola vai se abrindo. No botão em pré-antese, a corola já estava totalmente aberta. O início da antese ocorreu no início da manhã (por volta das 7:00h), quando o estigma estava receptivo e as anteras apresentavam coloração amarelo-esverdeada e os frisos longitudinais alaranjados. Ao longo da manhã, os frisos longitudinais das anteras liberaram odor, os poros se abriam e o pólen se tornava disponível, sendo liberado apenas por vibração. O estigma permanecia receptivo por todo o dia. Ao final da tarde, o odor não era mais perceptível e os frisos adquiriam coloração amarronzada. A antese teve duração de $c a .12$ horas. Na manhã do segundo dia, a maioria das flores já havia perdido os estames ou eles estavam amarronzados e senescentes e, em seguida, as pétalas começavam a cair. Na manhã do terceiro dia, o pistilo e o cálice persistiam, podendo ter persistido também a pétala de maior tamanho.

Quanto ao sistema reprodutivo a espécie não é apomítica (Tab. 1). Não houve formação de frutos a partir dos tratamentos autopolinização espontânea e manual com pólen proveniente dos três tamanhos de antera $\left(\mathrm{n}_{\text {total }}=137 ; \mathrm{n}_{\text {antera } \mathrm{P}}=39, \mathrm{n}_{\text {antera } \mathrm{M}}=46, \mathrm{n}_{\text {antera G }}=\right.$ 52) (Tab. 1). A forma em foice dos pistilos abortados foi a mesma da observada nas flores em antese, independentemente do momento do aborto. Os pistilos de polinização cruzada após cinco dias já adquiriram forma ereta. $\mathrm{O}$ acompanhamento do desenvolvimento de 66 pistilos autopolinizados por até dez dias após o cruzamento mostrou que $91 \%$ destes abortaram entre cinco a nove dias após o autocruzamento. No tratamento de polinização cruzada, não houve diferença entre a produção de frutos $(\mathrm{F})$ dos cruzamentos com pólen dos três tamanhos de antera $\left[X^{2}=0,94 ; \mathrm{p}=0,62 \mathrm{~F}_{\mathrm{pc}}=\mathrm{F}_{\text {total }}=0,53(55 / 104) ; \mathrm{F}_{\text {anteraP }}=\right.$ $\left.0,59(23 / 39), \mathrm{F}_{\text {antera }}=0,50(14 / 28), \mathrm{F}_{\text {antera }}=0,49(18 / 37)\right]$. Desta forma, esses dados foram agrupados (Tab. 1). Tubos polínicos provenientes de tratamentos de autopolinização manual e de polinização cruzada manual foram observados penetrando no ovário ou nos óvulos em flores fixadas 12 horas e 24 horas após a polinização (Fig. 1c-e). Não foram observadas diferenças aparentes na taxa de crescimento de tubos polínicos provenientes de autopolinização e polinização cruzada manual, nem de tratamentos com pólen proveniente dos três diferentes tamanhos de anteras. Não foi observado, através microscopia de fluorescência, desenvolvimento dos óvulos autopolinizados e de polinização cruzada nos pistilos fixados nos intervalos de tempo de 24, 48 e $72 \mathrm{~h}$.

A produção de frutos após polinização cruzada foi significativamente maior que em condições naturais (Tab. 2). No tratamento polinização cruzada, não houve diferença entre o número de óvulos $(\mathrm{H}=0,43, \mathrm{p}=0,81)$, de sementes desenvolvidas $(\mathrm{H}=0,10, \mathrm{p}=0,95)$, de sementes abortadas $(H=1,69, p=0,43)$, fecundidade $(H=0,18$, $\mathrm{p}=0,92)$ e taxa de aborto $(\mathrm{H}=1,45, \mathrm{p}=0,49)$ dos cruzamentos com pólen dos três tamanhos de antera. Esses dados foram agrupados e comparados às condições naturais (Tab. 2). A fecundidade no tratamento polinização cruzada foi significativamente maior em relação às condições naturais e a taxa de aborto não apresentou diferença (Tab. 2).

Os visitantes florais observados foram fêmeas de abelhas (Hymenoptera: Apoidea), que coletaram pólen. A coleta teve início por volta das 8:00h, permaneceu intensa até o início da tarde e diminuiu a partir das 14:00h. Foram consideradas pilhadoras abelhas pequenas $(<12 \mathrm{~mm})$ que durante a visita não contatavam o estigma e polinizadoras as abelhas de tamanho médio e grande $(\geq 12 \mathrm{~mm})$ que contatavam o estigma, como Euglossa iopoecila. Das quatorze espécies coletadas, $64 \%$ foram 

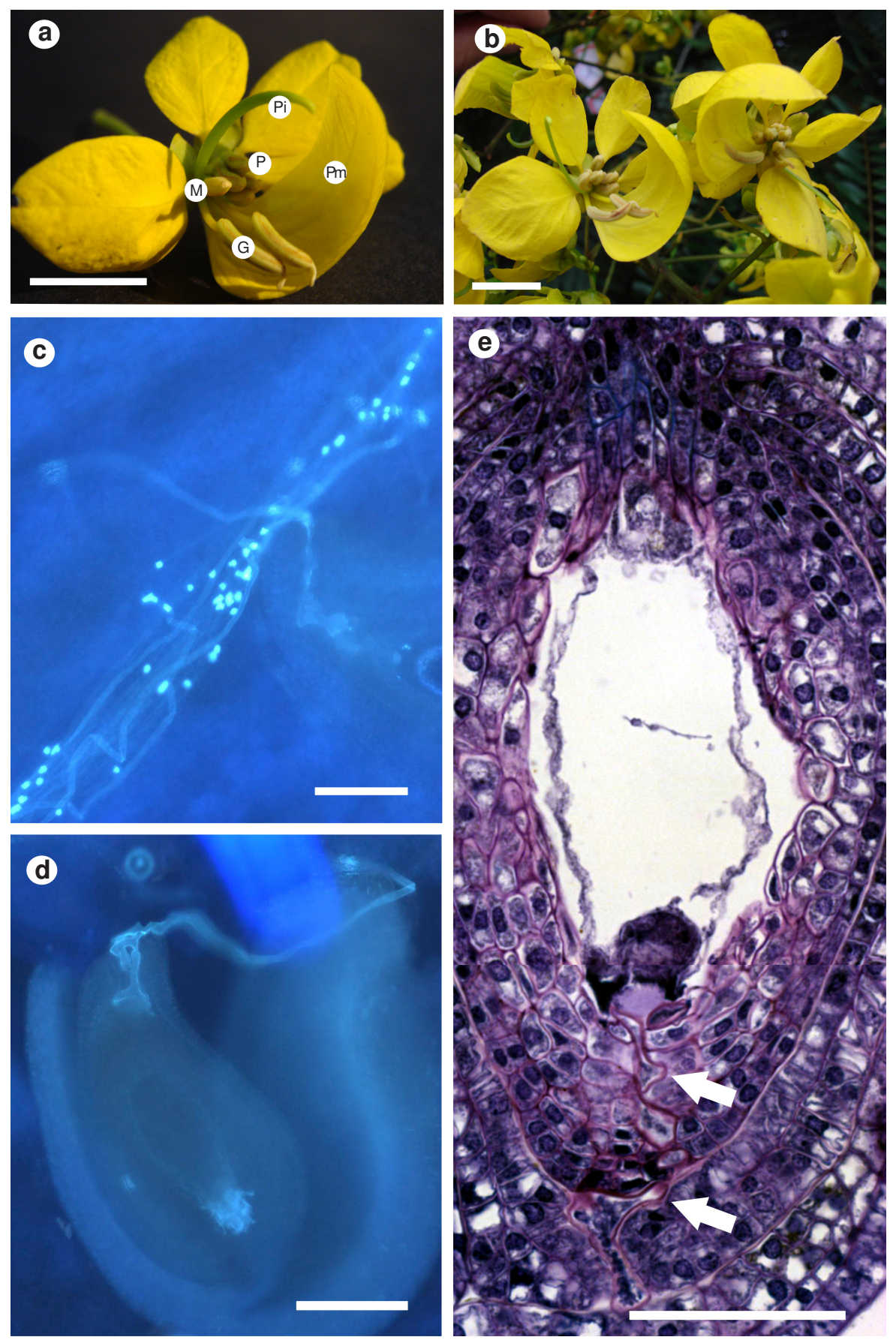

Figura 1 - Flor de Senna multijuga e crescimento de tubos polínicos - a. disposição das peças florais: pétala maior (Pm) e anteras grandes $(\mathrm{G})$ opostas ao pistilo (Pi) e ao estame médio $(\mathrm{M})$. Estames pequenos no centro da flor (P); b. inflorescência com flores dos dois morfos. c. penetração de tubos no ovário após autopolinização manual com pólen proveniente de antera pequena; d. tubo polínico penetrando em óvulo após autopolinização manual com pólen proveniente de antera grande; e. corte longitudinal de óvulo após autopolinização manual mostrando tubo polínico atravessando o tegumento e o nucelo (setas). Barras: a-b = 1cm, c-e $=100 \mu \mathrm{m}$.

Figure 1 - Flower of Senna multijuga and pollen tube growth - a. arrangement of floral parts: Major petal (pm) and large anthers (G) in opposition to the pistil $(\mathrm{Pi})$ and the medium stamen $(\mathrm{M})$. Small stamens in the center of the flower $(\mathrm{P})$; b. inflorescence with flowers of both morphs; c. penetration of tubes into the ovary 12 hours after manual self-pollination with pollen from a small anther; d. pollen tubes penetrating the ovary 12 hours after manual self-pollination with pollen from a major anther; e. longitudinal section of an ovule 24 hours after manual self-pollination showing pollen tube crossing the tegument and the nucellus (arrows). Bars: $\mathrm{a}-\mathrm{b}=1 \mathrm{~cm}, \mathrm{c}-\mathrm{e}=100 \mu \mathrm{m}$. 
Tabela 2 - Produção de frutos (porcentagem do número de frutos/número de flores), número de sementes desenvolvidas, de sementes abortadas e de óvulos, fecundidade (sementes desenvolvidas/óvulos) e taxa de aborto (sementes abortadas/ óvulos) de Senna multijuga, após polinização cruzada e em condições naturais. Dados são média \pm desvio padrão (exceto produção de frutos em \%).

Table 2 - Production of fruits (percentage of fruit number/flower number), number of seeds developed, seeds aborted and ovules, fecundity (seeds developed/ovules), and abortion rate (seeds aborted/ovules) of Senna multijuga after cross-pollination and natural conditions. Data are mean \pm standard deviation (except for fruit production in \%).

\begin{tabular}{lccll}
\hline & Polinização cruzada & Controle & Estatística & p \\
\hline Produção de frutos $(\%)$ & $53(55 / 104)$ & $14(13 / 90)$ & $X^{2}=31,32$ & $<0,0001$ \\
Sementes desenvolvidas & $31,44 \pm 6,57$ & $27,29 \pm 7,20$ & $\mathrm{U}=27,0$ & 0,18 \\
Sementes abortadas & $3,61 \pm 3,52$ & $6,47 \pm 2,68$ & $\mathrm{U}=22,0$ & 0,09 \\
Óvulos & $39,28 \pm 2,59$ & $45,07 \pm 5,09$ & $\mathrm{U}=11,0$ & 0,01 \\
Fecundidade & $0,80 \pm 0,16$ & $0,60 \pm 0,09$ & $\mathrm{U}=8,0$ & 0,01 \\
Taxa de aborto & $0,09 \pm 0,09$ & $0,15 \pm 0,07$ & $\mathrm{U}=25,0$ & 0,14 \\
\hline
\end{tabular}

classificadas como polinizadores, pois foi possível observar o contato do corpo na região estigmática durante a visita (Tab. 3). Essas abelhas se aproximavam da flor e se prendiam pela mandíbula e pernas dianteiras. A vibração produzida pelas abelhas durante o vôo era transmitida às anteras promovendo a liberação do pólen ( $c f$. Buchman 1983). As partes ventrais do tórax e do abdômen ficavam sobre as anteras de tamanho pequeno e a lateral do tórax e do abdômen ficavam próximas às outras anteras. As espécies de polinizadores mais freqüentes diferiram em relação ao comportamento de transferência de pólen entre as visitas. Bombus morio permanecia pairado e transferia o pólen do tórax e do abdômen para a corbícula, com auxílio das pernas dianteiras e medianas, enquanto as espécies de Centridini se prendiam com as mandíbulas nas estruturas florais e transferiam o pólen do tórax e do abdômen para as escopas das pernas traseiras com auxílio das pernas dianteiras e medianas. De maneira geral, as visitas duravam até três segundos. As abelhas visitaram diversas flores numa mesma inflorescência e diversas inflorescências de um mesmo indivíduo antes de deixarem a árvore.

\section{Discussão}

Senna multijuga apresenta morfologia floral semelhante à de outras espécies do gênero, por exemplo, pela assimetria da corola e androceu (Marazzi et al. 2006, 2007, 2008). As peculiaridades das estruturas florais em Senna, como diferentes tamanhos de estames e diferenças de orientação dos poros das anteras, provavelmente evoluíram em relação à polinização por vibração (Marazzi et al. 2007). A diversidade e maior especialização das anteras abaxiais em relação aos estames medianos podem estar relacionadas à divisão funcional das anteras, em alimentação e polinização, e ao comportamento de coleta de pólen pelas abelhas (Gottsberger \& Silberbauer-Gottsberger 1988; Silva et al. 2002; Marazzi et al. 2007). Contudo, não está claro se esta divisão implica em restrições reprodutivas ao pólen proveniente de cada morfo (Marazzi et al. 2007). Dados sobre a viabilidade e fertilidade do pólen proveniente de cada morfo de antera mostram que $S$. multijuga não apresentou restrição para a reprodução, semelhante a $S$. australis (Vell.) H.S. Irwin \& Barneby (Silva et al. 2002), porém, outras espécies do gênero a apresentaram, tais como $S$. corymbosa (Lam.) H.S. Irwin \& Barneby e S. sylvestris (Vell.) H.S. Irwin \& Barneby (Carvalho \& Oliveira 2003; Laporta 2005).

A orientação dos poros afeta diretamente a direção de liberação do pólen e a assimetria da corola tem um papel importante durante a polinização (Marazzi et al. 2007, 2008). Na flor de $S$. multijuga, a pétala maior é assimétrica, falcadoovada e côncava (Guimarães et al. 1984). A posição e a concavidade da pétala maior de $S$. multijuga parece ser uma característica importante para direcionar o pólen liberado das anteras grandes para a parte lateral e dorsal do corpo das abelhas e reduzir a perda de pólen, enquanto o pólen das anteras pequenas é liberado diretamente na região ventral do corpo das abelhas. A enantiostilia é outro aspecto da assimetria floral de S. multijuga que associado a heteranteria contribui na polinização. Jesson e Barrett (2005) sugerem que a deflexão das 
Tabela 3 - Espécies de abelhas (Hymenoptera: Apoidea) visitantes das flores de Senna multijuga. Legenda: Po = polinizador e $\mathrm{Pi}=$ pilhador.

Table 3 - Bees species (Hymenoptera: Apoidea) visitors of flowers of Senna multijuga . Captions: $\mathrm{Po}=$ pollinator and $\mathrm{Pi}=$ theft.

\begin{tabular}{lcc}
\hline Família & Espécie & Função \\
Tribo & & \\
\hline
\end{tabular}

\section{Apidae}

Euglossini

Bombini

Centridini

Xylocopini

Halictidae

Augochlorini
Euglossa iopoecila Dressler, 1982

$\mathrm{Pi}$

Bombus (Fervidobombus) brasiliensis Lepeletier, 1836

Po

Bombus morio Swederus, 1787

Po

Epicharis flava Friese, 1900

Po

Epicharis dejeanii Lepeletier, 1841

Po

Centris similis Fabricius, 1804

Po

Centris (Melacentris) cf. discolor Smith,1874

Po

Centris (Melacentris) cf. conspersa Mocsáry, $1899 \quad$ Po

Centris varia Erichson, 1848

Po

Melipona rufiventris Lepeletier, 1836

$\mathrm{Pi}$

Melipona sp.

Pi

Po

Xylocopa subcyanea Pérez, 1901

$\mathrm{Pi}$ anteras grandes opostas ao pistilo pode aumentar a precisão de transferência de pólen cruzado e reduzir a interferência do estigma e das anteras dentro ou entre flores da mesma planta. Desta forma, a distinção dos visitantes florais de $S$. multijuga em pilhadores e polinizadores é determinada pela combinação das características florais e a relação de tamanho com o corpo das abelhas. Os polinizadores de $S$. multijuga na área de estudo pertencem aos mesmos gêneros (Xylocopa, Centris, Bombus e Epicharis) dos polinizadores de outras espécies do gênero (Gottsberger \& Silberbauer-Gottsberger 1988; Silva et al. 2002; Carvalho \& Oliveira 2003; Laporta 2005; Pinheiro \& Sazima 2007). Entretanto, das nove espécies de polinizadores de $S$. multijuga no Itatiaia, apenas três (Bombus morio, Epicharis flava e Xylocopa frontalis) atuaram também como polinizadores desta espécie em área costeira em Ubatuba, São Paulo (Pinheiro \& Sazima 2007), a qual dista $c a .110 \mathrm{~km}$ do PNI. A composição de polinizadores de uma planta pode variar ao longo de sua área de ocorrência (Carvalho \& Oliveira 2003; Devoto et al. 2006) e isto pode refletir tanto divergências entre a distribuição geográfica da planta e dos polinizadores, como diferenças nas escolhas de forrageamento dos polinizadores, em resposta à composição e à fenologia das espécies de cada área.

Senna multijuga não formou frutos por autopolinização, o que em geral é tido como indicativo da presença de sistema de autoincompatibilidade. $\mathrm{O}$ crescimento de tubos polínicos no ovário e a penetração nos óvulos de pistilos autopolinizados indicam que não se trata de sistema de autoincompatibilidade gametofítico clássico (Gibbs 1990). Assim, o desenvolvimento de frutos após autopolinização pode estar sendo impedido por mecanismo de autoincompatibilidade de ação tardia (LSI). O termo LSI é utilizado para classificar mecanismos de rejeição diferentes e com origem independente, que essencialmente partilham a característica de não se enquadrarem nos mecanismos de autoincompatibilidade tradicionais (Seavey \& Bawa 1986). Os mecanismos de ação tardia são identificados basicamente pela inibição de tubos na camada do nucelo do óvulo ou a inibição da fusão dos gametas masculinos com a oosfera e núcleos polares nos casos de rejeição prezigótica; e pelo aborto dos óvulos em 
estágio inicial de desenvolvimento ou a degeneração do endosperma nos casos de rejeição pós-zigótica (Seavey \& Bawa 1986). Apesar disso, nem todos os registros de LSI em Fabaceae tem diferenciado os mecanismos de rejeição em pré- ou pós-zigótica. Evidências para a rejeição pós-zigótica podem ser observadas em várias espécies da família, assim como, em espécies de Bignoniaceae (Bittencourt et al. 2003; Bittencourt \& Semir 2005, 2006). Em Hymenaea stigonocarpa Mart. ex Hayne, isso se deu pela evidência de zigoto sem divisões com núcleos do endosperma (Gibbs et al. 1999), em Caesalpinia calycina Benth., pela evidência de penetração do tubo polínico no saco embrionário (Lewis \& Gibbs 1999), além de outras evidências para Dalbergia retusa Hemsl., Dipteryx panamensis (Pittier) Record \& Mell e Myrospermum frutescens Jacq. (Seavey \& Bawa 1986). Por outro lado, existe uma evidência para rejeição prezigótica em Acacia retinodes Schltdl. (Kenrick et al. 1986).

A ausência de frutos por autopolinização em espécies descritas com LSI pós-zigótica pode ser igualmente atribuída à depressão por endogamia, o que torna complicado diferenciar a presença de um mecanismo de autoincompatibilidade dos efeitos da depressão por endogamia (Seavey \& Bawa 1986; Sage et al. 1994). Segundo Seavey \& Bawa (1986), a autoincompatibilidade de ação tardia pode ser distinguida da depressão por endogamia por três critérios, a saber: 1) a rejeição por ação tardia ocorreria em um momento único, causando o aborto dos embriões em um estágio de desenvolvimento uniforme enquanto na depressão por endogamia, o acúmulo de alelos deletérios causaria o aborto progressivo de embriões em diferentes estágios de desenvolvimento; 2) na ação tardia, a maioria dos indivíduos da população deve ser autoestéril; 3) a ocorrência de autoincompatibilidade em espécies relacionadas indicaria a segregação de alelos de incompatibilidade dentro da família. O aborto de pistilos autopolinizados após um curto período de tempo, a ausência de desenvolvimento inicial dos óvulos autopolinizados e ausência de frutos nos cinco indivíduos que foram autopolinizados em S. multijuga sugerem a presença de um sistema de autoincompatibilidade de ação tardia.

Todavia, estudos que sugerem LSI em leguminosas não descartam a possibilidade de ocorrência de depressão por endogamia (Gibbs et al. 1999; Freitas \& Oliveira 2002). Outra evidência que os efeitos da depressão por endogamia podem imitar um mecanismo de autoincompatibilidade foi demonstrada em Platypodium elegans Vogel com o uso de marcadores moleculares utilizando progênies em diferentes estágios de desenvolvimento (embriões abortados, sementes maduras e plântulas) (Hufford \& Hamrick 2003). Neste estudo, houve diminuição da progênie autofertilizada ao longo dos estágios (expressa pelo aumento da heterozigosidade observada a cada nível) e a maior expressão da depressão por endogamia se deu entre o estágio de semente madura e o estabelecimento da plântula. Nesses casos, análises de progênies em diferentes estágios de desenvolvimento seriam mais eficientes do que testes de polinização controlados para responder tal questão.

As duas populações de $S$. multijuga estudadas por Ribeiro \& Lovato (2004), no estado de Minas Gerais, apresentaram diferentes taxas de cruzamento $\left(\mathrm{t}_{\mathrm{m}}=0,84\right.$ na área de pastagem fora do Parque e $\mathrm{t}_{\mathrm{m}}=0,54$ na área de borda). A média das populações $\left(\mathrm{t}_{\mathrm{m}}=0,69\right)$ e a menor taxa de cruzamento são inferiores às taxas registradas para a maioria das espécies arbóreas neotropicais com predomínio de cruzamento cruzado(Dick 2001; Quesada et al. 2004; Mathiasen et al. 2007; Borges et al. 2008). Portanto, Ribeiro \& Lovato (2004) concluem que seus resultados sugerem a ocorrência de autofecundação em S. multijuga, o que compromete a existência de LSI nesta espécie, sugerida pelos nossos resultados no Itatiaia.

Além disso, os resultados de Ribeiro \& Lovato (2004) apontam que S. multijuga deve apresentar sistema de cruzamento misto, o que pode refletir depressão endogâmica prematura e não necessariamente um mecanismo de LSI em relação ao aborto de frutos autopolinizados na espécie. Apenas por meio de testes de polinização é difícil concluir qual é o processo (endogamia ou mecanismo LSI) que atua para impedir a formação de frutos autopolinizados. De um lado, os níveis variáveis de alogamia entre populações mineiras de Senna multijuga sugerem existência de depressão por endogamia, mas por outro lado, o aborto uniforme de pistilos autopolinizados entre indivíduos na população do Itatiaia sugere LSI.

Em muitas espécies, os mecanismos de autoincompatibilidade não se expressam totalmente, ou seja, parte dos autocruzamentos produz frutos com sementes (Bawa 1974; Zapata \& Arroyo 1978; Sage et al. 2001). A expressão da autoincompatibilidade pode variar em função da composição de alelos, expressão de genes modificados, composição da carga de pólen, condições ambientais (e.g., temperatura) e condições internas do pistilo, tais como idade da flor, época do ano e presença de frutos em desenvolvimento (revisão em Stephenson et al. 2000). Gibbs et al. (2004) verificaram que a produção de sementes de Ceiba chodatii (Hassl.) Ravenna é inferior em frutos de cruzamentos com carga de pólen mista (auto e cruzado) em relação à carga de pólen cruzado. Neste 
estudo, os autores sugerem que a polinização natural é resultante da mistura de pólen (auto e cruzado) que chega ao estigma e que em espécies com LSI, essa carga de pólen mista pode resultar em uma proporção de sementes de autofertilização. Assim, esses autores predizem que espécies com LSI podem apresentar sistema de cruzamento misto. Outro aspecto relacionado a esta discussão é a variação da expressão da autoincompatibilidade entre populações de uma espécie (Sage et al. 2001; ver também Barrett et al. 1989 e Pailler \& Thompson 1997 para heterostilia), esperada em particular para certas circunstâncias, como em populações com tamanhos diferentes e nos limites geográficos de distribuição da espécie (Fausto et al. 2001; Stone et al. 2006). Desta forma, apesar dos resultados de Ribeiro \& Lovato (2004) sugerirem sistema de cruzamento misto e efeito de endogamia, isto não exclui a possibilidade de existir um mecanismo de incompatibilidade de ação tardia combinado, desde que parcial, em que sua expressão varie tanto por diferenças entre a composição da carga de pólen transportada pelos polinizadores como por diferenças entre populações da espécie.

$\mathrm{O}$ aumento significativo da produção de frutos e da fecundidade após polinização cruzada manual em relação às condições naturais pode indicar limitação nos níveis de polinização natural (Stephenson 1981; Burd 1995). Tal limitação poderia ser pela escassez de polinizadores e/ou pelo transporte inadequado de pólen compatível, visto que $30 \%$ das espécies visitantes apresentaram comportamento pilhador. Além disso, os visitantes legítimos visitam várias flores no mesmo indivíduo, o que reduz o fluxo de pólen e favorece a geitonogamia. Contudo, a produção de frutos $(52 \%)$ e a fecundidade $(0,80) \mathrm{em}$ S. multijuga após a polinização cruzada manual foram inferiores aos seus potenciais máximos. Isto se reflete em uma quantidade muito superior de flores que de frutos produzidos e indica que outros fatores podem atuar concomitantemente à limitação polínica, tais como a limitação por recursos, que é tida como um dos fatores mais importantes na regulação da produção de frutos e sementes nas plantas (Stephenson 1981). No contexto da seleção sexual, o sucesso reprodutivo masculino provavelmente é limitado pela capacidade dos gametas masculinos garantirem o acesso aos gametas femininos, enquanto o sucesso feminino é provavelmente limitado pela capacidade de prover recursos para os óvulos e embriões (Burd 1994). Bawa e Webb (1984) sugerem que variações nas razões fruto/flor e semente/óvulo entre espécies ocorrem de acordo com diferenças na intensidade da seleção sexual mais do que na disponibilidade de polinizadores e recursos. Nesse sentido, a seleção para aumentar a dispersão de pólen seria o principal fator na produção de flores excedentes de $S$. multijuga, enquanto o sucesso feminino parece ser regulado tanto pela limitação polínica como pela limitação por recursos.

Em resumo, embora nossos resultados não permitam concluir que Senna multijuga apresenta mecanismo de autoincompatibilidade, os testes de polinização controlada (ausência de frutos por autopolinização e aumento da frutificação e fecundidade após suplementação de pólen cruzado) demonstram que sua reprodução é dependente da transferência de pólen entre indivíduos realizada pelos polinizadores.

\section{Agradecimentos}

A Paulo Eugênio A.M. Oliveira e Alexandra P. Fernandez as sugestões em uma versão prévia e aos dois revisores anônimos as críticas e sugestões; a Leila Nunes Morgado e Maria Cristina Gaglianone a identificação das abelhas; a Victor Silvestre e Leandro Silva o auxílio no trabalho de campo; a Elaine Z. Souza, Rogério C. Figueiredo e Karen L.G. De Toni o auxílio na preparação e análise de material anatômico, respectivamente. Este estudo foi financiado pela Petrobrás (Programa Mata Atlântica - JBRJ). LF agradece ao CNPq a concessão de bolsa, modalidade PQ.

\section{Referências}

Arroyo, M.T.K. 1981. Breeding systems and pollination biology in Leguminosae. In: Polhill, R.M. \& Raven, P.H. Advances in Legume Systematics Part 2. Royal Botanical Garden, Kew. Pp. 723-769.

Ashton, P.S. 1969. Speciation among tropical forest trees: some deduction in the light of recent evidence. Biological Journal of the Linnean Society 1: 155-196.

Barrett, S.C.H.; Morgan, M.T. \& Husband. 1989. The dissolution of a complex genetic polymorphism: the evolution of self-fertilization in trustylous Eichornia paniculata (Pontederiaceae). Evolution 43: 1398-1416.

Barrett, S.C.H.; Jesson, L.K. \& Baker, A.M. 2000. The evolution and function of stylar polymorphism in flowering plants. Annals of Botany 85(Supp.A): 253-265.

Bawa, K.S. 1974. Breeding systems of tree species of lowland tropical community. Evolution 28: 85-92.

Bawa, K.S. \& Webb, C.J. 1984. Flower, fruit and seed abortion in tropical forest trees: implications or the evolution of paternal and maternal reproductive patterns. American Journal of Botany 71: 736-751. 
Bawa, K.S.; Perry, D.R. \& Beach, J.H. 1985a. Reproductive biology of tropical lowland rain forest trees. I. Sexual systems and incompatibility mechanisms. American Journal of Botany 72: 331-345.

Bawa, K.S.; Bullock, S.H.; Perry, D.R.; Coville, R.E. \& Grayum, M.H. 1985b. Reproductive biology of tropical lowland rain forest trees. II. Pollination systems. American Journal of Botany 72: 346-356.

Bittencourt Jr., N.S.; Gibbs, P.E. \& Semir, J. 2003. Histological study of post-pollination events in Spathodea campanulata Beauv. (Bignoniaceae), a species with late-acting self-incompatibility. Annals of Botany 91: 827-834.

Bittencourt Jr., N.S. \& Semir, J. 2005. Late-acting selfincompatibility and other breeding systems in Tabebuia (Bignoniaceae). International Journal of Plant Sciences 166: 493-506.

Bittencourt Jr., N.S. \& Semir, J. 2006. Floral biology and late-acting self-incompatibility in Jacaranda racemosa (Bignoniaceae). Australian Journal of Botany 54: 315-324.

Borges, L.A.; Sobrinho, M.S. \& Lopes, A.V. 2008. Phenology, pollination, and breeding system in the threatened tree Caesalpinia echinata Lam. (Fabaceae), and a review of studies on the reproductive biology in the genus. Flora 204: 111-130.

Buchman, S.L. 1983. Buzz pollination in angiosperms. In: Jone, C.E. \& Little, R.J. Handbook of experimental pollination biology. Scientific \& academic editions, New York. Pp. 73-113.

Burd, M. 1994. Bateman's principle and plant reproduction: the role of pollen limitation in fruit and seed set. Botanical Review 60: 83-139.

Burd, M. 1995. Ovule packing in stochastic pollination and fertilization environments. Evolution 49: 100-109.

Carvalho, D.A. \& Oliveira, P.E. 2003. Biologia reprodutiva e polinização de Senna sylvestris (Vell.) H.S. Irwin \& Barneby (Leguminosae, Caesalpinioideae). Revista Brasileira de Botânica 26: 319-328.

Cruden, R.W. 1977. Pollen-ovule ration: a conservative indicator of breading systems in flowering plants. Evolution 31: 32-46.

Dafni, A. 1992. Pollination ecology: a practical approach. Oxford University Press, New York. 250p.

Devoto, M.; Montaldo, N.H. \& Medan, D. 2006. Mixed hummingbird: Long-proboscid-fly pollination in 'ornithophilous' Embothrium coccineum (Proteaceae) along a rainfall gradient in Patagonia, Argentina. Austral Ecology 31: 512-519.

Dick, C.W. 2001. Genetic rescue of remmant tropical trees by alien pollinator. Proceedings of the Royal Society of London (B) 268: 2391-2396.

Fausto, J.A.; Eckhart, V.M. \& Geber, M.A. 2001. Reproductive assurance and the evolutionary ecology of self-pollination in Clarkia xantiana (Onagraceae). American Journal of Botany 88: 1794-1800.
Fedorov, A.A. 1966. The structure of the tropical rain forest and speciation in the humid tropics. Journal of Ecology 54: 1-11.

Frankie, G.W.; Haber, W.A.; Opler, P.A. \& Bawa, K.S. 1983. Characteristics and organization of the large bee pollination system in the Costa Rican dry forest. In: Jones, C.E. \& Little, R.J. Handbook of experimental pollination biology. Van Nostrand Reinhold, New York. Pp. 411-447.

Freitas, C.V. \& Oliveira, P.E. 2002. Biologia reprodutiva de Copaifera langsdorffii Desf. (Leguminosae, Caesalpinioideae). Revista Brasileira de Botânica 25: 311-321.

Gerrits, P.O. \& Smid, L. 1983. A new, less toxic polimerization system for the embedding og soft tissues in glicol methacrilate and subsequent preparing of serial sections. Journal of Microscopy 132: 81- 85.

Gibbs, P. 1990. Self-incompatibility in flowering plants: a neotropical perspective. Revista Brasileira de Botânica 13: 125-136.

Gibbs, P. \& Sassaki, R. 1998. Reproductive biology of Dalbergia miscolobium Benth. (LeguminosaePapilionoideae) in SE Brazil: the effects of pistillate sorting on fruit-set. Annals of Botany 81: 735-740.

Gibbs, P.; Oliveira, P.E. \& Bianchi, M.B. 1999. Postzygotic control of selfing in Hymenaea stigonocarpa (Leguminosae-Caesalpinioideae), a bat-pollinated tree of the Brazilian cerrados. Interntional Journal of Plant Sciences 160: 72-78.

Gibbs, P.; Bianchi, M.B. \& Taroda Ranga, N. 2004. Effects of self-, chase and mixed self/cross-pollinations on pistil longevity and fruit set in Ceiba species (Bombacaceae) with late-acting self-incompatibility. Annals of Botany 94: 305-310.

Goldenberg, R. \& Varassin, I.G. 2001. Sistemas reprodutivos de espécies de Melastomataceae da Serra do Japi, Jundiaí, São Paulo, Brasil. Revista Brasileira de Botânica 24: 283-288.

Gottsberger, G. \& Silberbauer-Gottsberger, I. 1988. Evolution of flower structure and pollination in neotropical Cassinae (Caesalpiniaceae) species. Phyton 28: 293-320.

Gribel, R.; Gibbs, P. \& Queiróz, A. L. 1999. Flowering phenology and pollination biology of Ceiba pentandra (Bombacaceae) in Central Amazon. Journal of Tropical Ecology 15: 247-263.

Guimarães, D.J.; Fontenelle, G.B. \& Oliveira, B.A.D. 1984. Anatomia do desenvolvimento floral de Cassia multijuga Rich. (Leguminosae-Caesalpinoideae). Rodriguésia 36: 73-80.

Hufford, K.M. \& Hamrick, J.L. 2003. Viability selection at three early life stages of the tropical tree, Platypodium elegans (Fabaceae, Papilionoideae). Evolution 57: 518-526.

Husband, B.C. \& Schemske, D.W. 1996. Evolution of the magnitude and timing of inbreeding depression in plants. Evolution 50: 54-70. 
Irwin, M.S. \& Barneby, R.C. 1982. The american Cassinae. Asynoptical revision of Leguminosae tribe Cassieae subtribe Cassinae in the New World. Memories of the New York Botanical Garden 35: 1-918.

Janzen, D.H. 1971. Euglossine bees as long-distance pollinators of tropical plants. Science 171: 203-205.

Jesson, L.K.; Kang J.; Wagner, S.L.; Barrett, S.C.H. \& Dengler, N.G. 2003. The development of enantiostyly. American Journal of Botany 90: 183-195.

Jesson, L.K. \& Barrett, S.C.H. 2005. Experimental tests of the function of mirror-image flowers. Biological Journal of the Linnean Society 85: 167-179.

Kenrick, J.; Kaul, V. \&Williams, E.G. 1986. Self-incompatibility in Acacia retinodes: site of pollen-tube arrest is the nucellus. Planta 169: 245-250.

Laporta, C. 2005. Floral biology and reproductive system of enantiostylous Senna corymbosa (Caesalpiniaceae). Revista de Biologia Tropical 53: 49-61.

Lewis, G. \& Gibbs, P. 1999. Reproductive biology of Caesalpinia calycina and C. pluviosa (Leguminosae) of the caatinga of north-eastern Brazil. Plant Systematics and Evolution 217: 43-53.

Marazzi, B.; Endress, P.K.; Queiroz, L.P. \& Conti, E. 2006. Phylogenetic relationships within Senna (Leguminosae, Cassinae) based on three chloroplast DNA regions: patterns in the evolution of floral symmetry and extrafloral nectaries. American Journal of Botany 93: 288-303.

Marazzi, B.; Conti, E. \& Endress, P.K. 2007. Diversity in anthers and stigmas in the buzz-pollinated genus Senna (Leguminosae, Cassinae). International Journal of Plant Science 168(4): 371-391.

Marazzi, B. \& Endress, P.K. 2008. Patterns and development of floral asymmetry in Senna (Leguminosae, Cassiinae). American Journal of Botany 95: 22-40.

Martin, F.N. 1959. Staining and observing pollen tubes in the style by means of fluorescence. Stain Technology 34: 125-128.

Mathiasen, P.; Rovere, A.E. \& Premoli, A.C. 2007. Genetic structure and early effects of inbreeding in fragmented temperate forests of a self-incompatible tree, Embothrium coccineum. Conservation Biology 21: 232-240.

Moraes, L.F.D., Assumpção, J.M., Pereira, T.S. \& Luchiari, C. 2006. Manual técnico para a restauração de áreas degradadas no estado do Rio de Janeiro. Instituto de Pesquisas Jardim Botânico do Rio de Janeiro, Rio de Janeiro. 84p.

Morim, M.P. 2006. Leguminosae arbustivas e arbóreas da Floresta Atlântica do Parque Nacional de Itatiaia, sudeste do Brasil: padrões de distribuição. Rodriguésia 57: 27-45.

Morim, M.P. \& Barroso, G.M. 2007. Leguminosae arbustivas e arbóreas da Floresta Atlântica do Parque Nacional de Itatiaia, sudeste do Brasil: subfamílias Caesalpinioideae e Mimosoideae. Rodriguésia 58: 423-468.
O'Brien, T.P., Feder, N. \& McCully, M.E. 1965. Polychromatic staining of plant cell walls by toluidine blue O. Protoplasma 59: 368-373.

Pailler, T. \& Thompson, J.D. 1997. Distyly and variation in heteromorphic incompatibility in Gaertnera vaginata (Rubiaceae) endemic to La Reunion Island. American Journal of Botany 84: 315-327.

Pinheiro, M. \& Sazima, M. 2007. Visitantes florais e polinizadores de seis espécies arbóreas de Leguminosae melitófilas na Mata Atlântica no Sudeste do Brasil. Revista Brasileira de Biociências 5(supl.1): 447-499.

Pires, J.P.A. \& Freitas, L. 2008. Reproductive biology of two tree species of Leguminosae in a Montane Rain Forest in Southeastern Brazil. Flora 203: 491-498.

Quesada, M.; Stoner, K.E.; Lobo, J.A.; Herrerías-Diego, Y.; Palácios-Guevara, C., Munguia-Rosas, M.A., Salazar, K.A.O. \& Rosas-Guerrero, V. 2004. Effects of forest fragmentation on pollinator activity and consequences for plant reproductive success and mating patterns in bat-pollinated bombacaceous trees. Biotropica 36: 131-138.

Ribeiro, R.A. \& Lovato, M.B. 2004. Mating system in a neotropical tree species, Senna multijuga (Fabaceae). Genetics and Molecular Biology 27: 418-424.

Rodrigues, R.S.; Flores, A.S.; Miotto, S.T.S. \& Baptista, L.R.M. 2005. O gênero Senna (Leguminosae, Caesalpinioideae) no Rio Grande do Sul, Brasil. Acta Botanica Brasilica 19(1): 1-16.

Sage, T.L.; Bertin, R.I. \& Williams, E.G. 1994. Ovarian and other late-acting self-incompatibility systems. In: Williams, E.G.; Clarke, A.E. \& Knox, R.B. (eds.). Genetic control of self-incompatibilit and reproductive development in flowering plants. Kluwer, Dordrecht. Pp 116-140.

Sage, T.L.; Griffin, S.R.; Pontieri, V.; Drobac, P.; Cole, W.W. \& Barrett, S.C.H. 2001. Stigmatic selfincompatibility and mating patterns in Trillium grandiflorum and Trillium erectum (Melanthiaceae). Annals of Botany 88: 829-841.

Sazima, M., Sazima, I \& De Carvalho-Okano, R.M. 1985. Biologia floral de Dalechampia stipulacea (Euphorbiaceae) e sua polinização por Euglossa melanotricha (Apidae). Revista Brasileira de Botânica 45(1/2): 85-93.

Seavey, S.R. \& Bawa, K.S. 1986. Late-acting selfincompatibility in Angiosperms. Botanical Review 52: 195-219.

Seavey, S.R. \& Carter, S.K. 1994. Self-Sterility in Epilobium obcordatum (Onagraceae). American Journal of Botany 81: 331-338.

Segadas-Vianna, F. \& Dau, L. 1965. Ecology of Itatiaia range, southeastern Brazil. II, Climates and altitudinal climatic zonation. Arquivos do Museu Nacional Rio de Janeiro 53: 31-53. 
Silva, A.L.G.; Ormond, W.T. \& Pinheiro, M.C.B. 2002. Biologia floral e reprodutiva de Senna australis (Vell.) Irwin \& Barneby (Fabaceae, Caesalpinioideae). Boletim do Museu Nacional 121: 1-11.

Statsoft, 2001. Statistica (data analysis software system). version 6.0. www.statsoft.com S. Tulsa, USA.

Stephenson, A.G. 1981. Flower and fruit abortion: proximate causes and ultimate functions. Annual Review of Ecology, Evolution and Systematics 12: 432-440.

Stephenson, A.G.; Good, S.V. \& Vogler, D.W. 2000. Interrelationships among inbreeding depression, plasticity in the self-incompatibility system, and the breeding system of Campanula rapunculoides $\mathrm{L}$. (Campanulaceae). Annals of Botany 85: 211-219.

Stone, J.L.; Sasuclark, M.A. \& Blomberg, C.P. 2006. Variation in the self-incompatibility response within and among populations of the tropical shrub Witheringia solanacea (Solanaceae). American Journal of Botany 93: 592-598.
Teixeira, L.A.G. \& Machado, I.C. 2000. Sistema de polinização e reprodução de Byrsonima sericea DC.(Malpighiaceae). Acta Botanica Brasilica 14: 347-357.

Torres, M.W. 2009. Biologia reprodutiva e polinização de Senna multijuga no Parque Nacional de Itatiaia e na Área de Proteção Ambiental da Serrinha do Alambari. Dissertação de Mestrado. IPJBRJ, Rio de Janeiro. 49p.

Veloso, H.P.; Rangel Filho, A.L.R. \& Lima, J.C.A. 1991. Classificação da vegetação brasileira, adaptada a um sistema universal. Instituto Brasileiro de Geografia e Estatística, Rio de Janeiro. 116p.

Zapata, T.R. \& Arroyo, K.M.T. 1978. Plant reproductive ecology of a secondary deciduous tropical forest in Venezuela. Biotropica 10: 221-230.

Zeisler, M. 1938. Über die Abgrenzung der eigentlichen Narbenfläche mit Hilfe von Reaktionen. Beith. Bot. Centralbl. 58: 308-318. 